\title{
Correlation between Demographic, Socio-economic, and Cancer-specific Factors with Quality of Life Scores among Newly-Diagnosed Cancer Patients of the Medical Oncology Clinics of the Philippine General Hospital Cancer Institute
}

\author{
Mary Ondinee U. Manalo and Corazon A. Ngelangel
}

Section of Medical Oncology, Department of Medicine, College of Medicine and Philippine General Hospital, University of the Philippines Manila

\begin{abstract}
Introduction. Over the last two decades, psychosocial research has explored the experience of cancer patients. This study evaluated if demographic, socio-economic and cancer-specific factors impact and correlate with quality of life (QoL) scores at the time of first consult of newly-diagnosed cancer patients seen at medical oncology clinics of the University of the PhilippinesPhilippine General Hospital (UP-PGH) Cancer Institute from 2012-2013.

Methods. Review of charts and interview with a pre-approved and validated questionnaire were done after informed consent. Age, gender, marital status, number of close friends, household income band, employment status, cancer site and stage were recorded. Outcomes were cancer-specific QoL EORTC QLQ-C30 questionnaire and generic QoL EQ5D questionnaire. Scores were correlated with demographic, socio-economic, and cancerspecific factors.
\end{abstract}

Results. 535 patients were included, 257 male and 278 female. Mean age was 52 years (SD 13.5 years; range20-92 years). Majority (28.7\%) belonged to income bracket P4,293$\mathrm{P} 8,583 /$ month. Majority were married $(74.31 \%)$ and unemployed (58.4\%). Top 5 cancers were colorectal (28.09\%), breast $(20.70 \%)$, head and neck (16.63\%), lung (9.97\%), lymphoma (7.94\%). According to EORTC QLQ-C30, physical functioning ( $p=0.0037)$ and cognitive functioning $(p=0.003)$ were significantly correlated with younger patients while role functioning $(p=0.04)$ and emotional functioning $(p=0.03)$ showed negative correlation with older patients. Fatigue was less in female patients $(p=0.0005)$ while being the household head $(p=0.0005)$ was

\footnotetext{
Corresponding author: Corazon A. Ngelangel, MD, MS, PhD Section of Medical Oncology

Department of Medicine

Philippine General Hospital

University of the Philippines Manila

Taft Avenue, Ermita, Manila 1000 Philippines

Tele/Fax: +632 5263775

Email: corazon.ngelangel@gmail.com
}

significantly correlated with increased fatigue. According to EQ5D, single patients ( $p=0.016$ ) had better mobility than the rest of patients. Having 5 family members significantly reported less pain $(p=0.038)$. Breast cancer patients had best QOL while bladder cancer patients had the worst QOL. As cancer stage increased, QOL decreased.

Conclusion. This is a first baseline study on self-reported QOL among newly-diagnosed Filipino cancer patients, an important relevant reference in the field of psychosocial issues among lowresourced cancer patients in the Philippines.

Key Words: QoL, Philippine cancer patients

\section{Introduction}

Cancer has been cited as the leading cause of mortality globally, accounting for $13 \%$ (or 7.4 million) of all deaths annually ${ }^{1}$ with $70 \%$ of these occurring in low and middle income countries. It is projected that mortality from cancer will increase significantly over the coming years with $~ 13$ million deaths per year worldwide expected by 2030. The trend is even more striking in Asia where the number of deaths per year in 2002 of 3.5 million is expected to increase to 8.1 million by $2020 .^{2}$ As the availability of medical technologies and treatments expands across regions, the economic burden of cancer treatments, not only to health systems but to individuals and their households, will inevitably become more pronounced. These impacts will be felt most strongly in socioeconomically disadvantaged groups particularly (although not exclusively) those in low and middle income countries where social safety nets, such as universal health insurance, are less likely to be present. A consequence of this is that such illness, particularly through the costs associated with its treatment and its impact on people's ability to work, can be a major cause of poverty.

During the last decade, the spectrum of endpoints used to evaluate medical treatments has widened. Physical, psychological, and social problems/symptoms related to disease or its treatment are now to a greater extent recognized as important outcomes. Aside from measurement of disease, quality of life (QoL) has been 
measured alongside disease response or progression, especially in clinical trials. QoL is a broad term without exact definition. It depends on a number of factors: support from friends and relatives, ability to work and interest in one's occupations, accommodation appropriate to expectations and, of course, health and other co-morbidities. In the field of most clinicians, by their training, concentrate attention on the somatic and tangible illness; the role of emotional disorder be it a reaction to the somatic illness or an independent factor, is often overlooked. ${ }^{1}$

It is generally accepted that data concerning the patients' well-being should be provided by the patients themselves. ${ }^{2,3}$ Standardized questionnaires for patient selfassessment have been developed and are used for that purpose in clinical research. It is critical that the validity and reliability of such measures be evaluated. ${ }^{4,5}$ The two most popularly used questionnaires to evaluate QoL in cancer patients are the EQ-5D and the EORTC QLQ-C30. These two questionnaires have been extensively validated and are currently used in clinical trials, population surveys, and measuring self-reported inequalities in healthcare.

Recent studies have identified baseline QoL as a prognostic factor of survival in cancer patients. ${ }^{6-8}$ There has been no attempt yet in the Philippines to correlate demographics and socio-economic factors with QoL scores among newly-diagnosed cancer patients to determine who among them are at risk to have poor quality of life during the initial visits, and thus would need additional care. It is in this light that this study was undertaken.

\section{Methods}

\section{Study Design}

This is a cross-sectional study of patients with first time diagnosis of cancer in the medical oncology clinics of the cancer institute of a tertiary hospital (UP-PGH).

\section{Participants}

Consecutive new patients consulting at the OPD were recruited and included in the study after informed consent. The patients were 18 years and older, with first time cancer diagnosis received in the last 12 weeks, aware of their new cancer diagnosis, conscious with sufficient cognitive capacity to give informed consent and complete an interview. The patients were excluded if they were participating in a clinical trial and if they had prior chemotherapy or radiotherapy that would affect their QOL.

\section{Measures}

After obtaining informed consent from the patients, a standardized questionnaire addressing patient characteristics was used. Data collected included items marital status, education, employment, household income, number of close friends, cancer site and cancer stage. Two health-related quality of life (HRQOL) questionnaires were used - the validated Tagalog or English versions of the European Organization for Research and Treatment of Cancer Study Group on Quality of Life (EORTC- QLQ-C30) and the European Quality of Life Group (EQ-5D).

The EORTC QLQ-C30 is a HRQOL questionnaire, developed by the European Organization on Research and Treatment of Cancer (EORTC) Study Group on QOL. ${ }^{9}$ The core questionnaire is intended to measure general aspects of HRQOL specific to cancer patients. EOTC QLQ-C30, version 3 , incorporates five functional scales on physical, role, cognitive, emotional, and social functioning, three symptom scales on fatigue, pain and nausea and vomiting, single items assessing dyspnea, insomnia, loss of appetite, constipation and diarrhea, one item assessing perceived financial impact and a global health status/QOL scale. Each status is scored in one of four categories: 1) 'not at all'; 2) 'a little'; 3) 'quite a bit'; 4) 'very much', with the exception of 'global QoL', which ranges from 1) 'very poor' to 7) excellent. ${ }^{10}$

EQ-5D is a short self-reported generic health-related QoL instrument that consists of two parts: a self-classifier and a visual analogue scale (VAS). It is a standardised measure of health status developed by the EuroQol Group in order to provide a simple, generic measure of health for clinical and economic appraisal. Applicable to a wide range of health conditions and treatments, it provides a simple descriptive profile and a single index value for health status that can be used in the clinical and economic evaluation of health care as well as in population health surveys. ${ }^{11}$ Mean scores are taken for this particular measure of QoL. EQ-5D is designed for self-completion by respondents and is ideally suited for use in postal surveys, in clinics, and in face-to-face interviews. It is cognitively undemanding, taking only a few minutes to complete. Instructions to respondents are included in the questionnaire. The EQ-5D 3 level version (EQ-5D-3L) was introduced in 1990. The EQ-5D-3L essentially consists of 2 pages - the EQ-5D descriptive system and the EQ visual analogue scale (EQ VAS). The EQ5D-3L descriptive system comprises the following 5 dimensions: mobility, self-care, usual activities, pain/discomfort and anxiety/depression. Each dimension has 3 levels: no problems, some problems, extreme problems. The respondent is asked to indicate his/her health state by ticking (or placing a cross) in the box against the most appropriate statement in each of the 5 dimensions. The EQ VAS records the respondent's self-rated health on a vertical, visual analogue scale where the endpoints are labelled 'best imaginable health state' and 'worst imaginable health state'. Mean scores are also taken for this particular measure of QoL. 


\section{Ethical Considerations}

The protocol was approved by the UP Ethics Committee. Informed consent was taken from all subjects prior any data collection; data confidentiality was practiced.

\section{Statistics}

For the quality of life analysis, a linear transformation to a '0-100' scale of the EORTC QLQ-C30 questionnaire was carried out according to the EORTC Scoring Manual. A higher mean score for functional scales and global QoL reflects a better level functioning, but a higher mean score for symptoms reflects more problems. In EQ-5D, combinations of each domain with their categories define a total of 243 health states.

Continuous variables were summarized as means while categorical variables were presented as frequencies. Mean scores and standard deviations (SD) were calculated on the summated scales and items. T-test was used to examine differences between subgroups and Mann-Whitney test was used to quantify these differences between two subgroups. Associations between two categorical variables were explored using chi-square test or Fisher's exact test whenever applicable. Kruskal-Wallis was used to test associations between 3 or more categories. Spearman rho was used for associations between two continuous variables. Shapiro-Wilk test was used to check normality of assumptions.

Stata Statistics Data Analysis Software version 12.0 was used to analyze data. All calculated p-values were two-sided and p-values less than 0.05 were considered statistically significant. A statistician from the University of the Philippines-Manila College of Public Health analysed data with the authors.

\section{Results}

Out of 550 possible newly-diagnosed participants (within 12 weeks of diagnosis) in the study, only 535 had complete data and returned the informed consent. These were included in the final analysis. None of the 535 participants had missing values. A reliability analysis (internal consistency) was performed for all levels of QoL, reaching satisfactory levels of Cronbach's alpha (0.9304).

\section{Baseline characteristics}

Five hundred thirty-five patients were recruited for the study, with a mean age of $52 \pm 13.5$ years. Majority of patients were in the 50-59 year range (31.03\%), female $(51.96 \%)$, married $(74.21 \%)$, high school graduate $(38.13 \%)$, and were working in the field of agriculture or fisheries
(37.57\%). Majority source of income came from salaries $(68.97 \%)$ while most fell in the Philippines' income bracket 2 according to NHES data $(28.41 \%)$. The details of the results are found in Table 1. The most common type of cancer was colorectal $(28.22 \%)$ while the most common stage was Stage 3 (36.45\%). Cancer-specific details are found in Table 2.

\section{Quality of Life by EORTC}

Table 3 shows the means of patient scores for QoL stratified according to age. Physical functioning $(p=0.0037)$ and cognitive functioning $(p=0.003)$ were positively correlated with the younger patients, while role functioning $(p=0.04)$ and emotional functioning $(p=0.03)$ correlated negatively with the older populations. Younger patients generally had better QoL scores than their older counterparts.

There was less fatigue among the female population in all age groups $(p=0.0005)$, the rest of the categories failed to show statistical significance with regard to gender. Also, fatigue was significantly correlated with marital status with single patients experiencing less fatigue than married $(p=0.0365)$ or separated $(p=0.0302)$ patients. Being the household head was also positively correlated with fatigue $(p=0.0114)$. Educational status was not correlated with any of the indicators of QoL. Table 4 shows the means for gender, marital status and educational attainment.

Presence of insurance, type of work, and income were not significantly correlated with QoL (Tables 4 and 5). Number of permanent household members was not significantly correlated with QoL. What was remarkable was that having extremes of friends (none and more than 10) were associated with poorer QoL scores while having around 5 close friends was significantly correlated with better scores.

\section{Quality of Life by EQ-5D}

A decreasing trend for mobility was shown as age increased $(p=0.001$ (Table 6). Single patients $(p=0.016)$ had better mobility than the rest of patients (Table 7). Pain was significantly correlated with the number of household members with patients having 5 family members significantly reporting less pain $(\mathrm{p}=0.038)$. Personal care was notably better among patients who belonged to the lower income bracket $(\mathrm{p}=0.002)$ (Table 8$)$. The rest of the categories for EQ5D with regard to usual activities (e.g. educational attainment, type of work) failed to reach statistical significance for demographics and socio-economic differences (Tables 9 and 10). 
Table 1. Demographics and socioeconomic factors, $n=535$

\begin{tabular}{|c|c|c|c|c|c|}
\hline Factors & $\mathbf{n}$ & $\%$ & Factors & $\mathrm{n}$ & $\%$ \\
\hline Age category (years) & & & Monthly income & & \\
\hline $20-29$ & 31 & 5.79 & Bracket 1: Php 0-4,292 & 112 & 20.93 \\
\hline $30-39$ & 60 & 11.21 & Bracket 2: Php 4,293-8,583 & 152 & 28.41 \\
\hline $40-49$ & 107 & 20.00 & Bracket 3: Php 8,584-12,875 & 96 & 17.94 \\
\hline $50-59$ & 166 & 31.03 & Bracket 4: Php 12,876-17,167 & 67 & 12.52 \\
\hline $60-69$ & 121 & 22.62 & Bracket 5: Php 17,167-21,458 & 34 & 6.36 \\
\hline $70-79$ & 42 & 7.85 & Bracket 6: Php 21,459-25,750 & 24 & 4.49 \\
\hline \multirow[t]{2}{*}{80 and above } & 8 & 1.50 & Bracket 7: Php 25,751-30,042 & 17 & 3.18 \\
\hline & & & Bracket 8: Php 30,043-34,333 & 7 & 1.31 \\
\hline Gender & & & Bracket 9: Php $>34,333$ & 26 & 4.86 \\
\hline Male & 257 & 48.04 & & & \\
\hline \multirow[t]{2}{*}{ Female } & 278 & 51.96 & Household head & & \\
\hline & & & Yes & 265 & 49.53 \\
\hline Civil status & & & No & 269 & 50.28 \\
\hline Single & 62 & 11.59 & & & \\
\hline Married & 397 & 74.21 & Number of Household members & & \\
\hline Widowed & 60 & 11.21 & 1 & 9 & 1.68 \\
\hline \multirow[t]{2}{*}{ Separated } & 16 & 2.99 & 2 & 50 & 9.35 \\
\hline & & & 3 & 91 & 17.01 \\
\hline Highest educational attainment & & & 4 & 92 & 17.20 \\
\hline No formal education & 7 & 1.31 & 5 & 107 & 20.00 \\
\hline Elementary & 135 & 25.23 & 6 & 88 & 16.45 \\
\hline High school & 204 & 38.13 & 7 & 42 & 7.85 \\
\hline Vocational & 46 & 8.60 & 8 & 29 & 5.42 \\
\hline \multirow[t]{2}{*}{ College } & 143 & 26.73 & 9 & 9 & 1.68 \\
\hline & & & $\geq 10$ & 18 & 3.37 \\
\hline \multicolumn{6}{|l|}{ Kind of work } \\
\hline Manager & 12 & 2.25 & \multicolumn{3}{|c|}{ Number of Household members $\leq 15$ years } \\
\hline Professional & 55 & 10.28 & 0 & 224 & 41.87 \\
\hline Clerical support worker & 21 & 3.93 & 1 & 158 & 29.53 \\
\hline Vendor & 26 & 4.86 & 2 & 72 & 13.46 \\
\hline Worker in the field of agriculture, fisheries and forestry & 201 & 37.57 & $\geq 3$ & 81 & 15.14 \\
\hline Craft and related trades & 58 & 10.84 & & & \\
\hline Factory worker & 32 & 5.98 & \multicolumn{3}{|c|}{ Number of Household members $\geq 65$ years } \\
\hline Domestic work & 40 & 7.48 & 0 & 379 & 70.84 \\
\hline None & 54 & 10.09 & 1 & 113 & 21.12 \\
\hline Retired with pension & 26 & 4.86 & 2 & 42 & 7.85 \\
\hline Retired without pension & 10 & 1.87 & $\geq 3$ & 1 & 0.19 \\
\hline Presence of insurance & & & Number of friends & & \\
\hline Yes & 39 & 7.29 & 0 & 181 & 33.83 \\
\hline \multirow[t]{2}{*}{ No } & 496 & 92.71 & 1 & 43 & 8.04 \\
\hline & & & 2 & 51 & 9.53 \\
\hline Source of income & & & 3 & 44 & 8.22 \\
\hline Crops & 31 & 5.79 & 4 & 19 & 3.55 \\
\hline Livestock & 15 & 2.80 & 5 & 39 & 7.29 \\
\hline Family business & 49 & 9.16 & 6 & 6 & 1.12 \\
\hline Salary & 369 & 68.97 & 7 & 3 & 0.56 \\
\hline Donations and gifts & 69 & 12.90 & 8 & 3 & 0.56 \\
\hline \multirow[t]{2}{*}{ Others } & 2 & 0.37 & 9 & 1 & 0.19 \\
\hline & & & $\geq 10$ & 145 & 27.10 \\
\hline
\end{tabular}


QoL Cancer Patients

Table 2. Distribution by cancer site and stage

\begin{tabular}{|c|c|c|}
\hline Type of cancer & $\mathbf{n}$ & $\%$ \\
\hline Colorectal & 151 & 28.22 \\
\hline Breast & 102 & 19.07 \\
\hline Mouth-pharynx & 89 & 16.64 \\
\hline Others & 68 & 12.71 \\
\hline Trachea, bronchus, lungs & 53 & 9.91 \\
\hline Lymphoma, myeloma & 43 & 8.04 \\
\hline Melanoma & 5 & 0.93 \\
\hline Prostate & 5 & 0.93 \\
\hline Bladder & 4 & 0.74 \\
\hline Esophagus & 4 & 0.74 \\
\hline Liver & 4 & 0.74 \\
\hline Skin & 4 & 0.74 \\
\hline Pancreas & 2 & 0.37 \\
\hline Cervix & 1 & 0.19 \\
\hline Cancer stage & $\mathrm{n}$ & $\%$ \\
\hline 1 & 33 & 6.17 \\
\hline 2 & 120 & 22.43 \\
\hline 3 & 195 & 36.45 \\
\hline 4 & 187 & 34.95 \\
\hline
\end{tabular}

Table 3. Means for EORTC QLQ-C30 functional scales, symptom scales and single items by age

\begin{tabular}{|c|c|c|c|c|c|c|c|c|c|c|c|c|c|c|}
\hline \multirow{2}{*}{ Scale } & \multicolumn{14}{|c|}{ Age range (Mean, SD) } \\
\hline & $20-29$ & SD & $30-39$ & SD & $40-49$ & SD & $50-59$ & SD & $60-69$ & SD & $70-79$ & SD & $\geq 80$ & SD \\
\hline QL2 & 57.53 & 23.11 & 54.64 & 19.19 & 59.96 & 20.62 & 57.47 & 20.17 & 53.78 & 20.38 & 46.03 & 18.15 & 51.04 & 24.57 \\
\hline PF2 & 74.84 & 32.19 & 77.11 & 24.68 & 80.12 & 19.34 & 80.56 & 22.12 & 75.65 & 25.19 & 62.38 & 28.05 & 76.67 & 18.85 \\
\hline RF2 & 65.05 & 33.71 & 66.11 & 35.25 & 67.45 & 32.08 & 70.18 & 30.52 & 68.32 & 31.36 & 53.57 & 38.71 & 58.33 & 39.84 \\
\hline EF & 67.74 & 26.33 & 70.00 & 22.45 & 72.51 & 19.60 & 70.28 & 21.64 & 71.69 & 22.06 & 67.85 & 21.6 & 62.5 & 23.14 \\
\hline $\mathrm{CF}$ & 84.95 & 22.91 & 85.83 & 17.58 & 86.92 & 19.56 & 88.35 & 17.26 & 84.43 & 18.97 & 76.98 & 20.81 & 75.00 & 21.82 \\
\hline SF & 75.81 & 34.92 & 69.17 & 30.04 & 74.14 & 30.39 & 74.19 & 29.04 & 78.92 & 28.11 & 74.98 & 28.34 & 81.25 & 24.29 \\
\hline FA & 20.43 & 26.46 & 25.37 & 28.72 & 19.11 & 21.08 & 20.88 & 20.13 & 22.68 & 25.67 & 74.60 & 24.67 & 23.61 & 17.25 \\
\hline NV & 4.30 & 10.51 & 9.16 & 19.98 & 5.14 & 14.37 & 5.02 & 11.38 & 5.23 & 11.79 & 30.42 & 18.08 & 8.33 & 23.57 \\
\hline PA & 30.11 & 33.99 & 35.00 & 31.39 & 31.78 & 27.81 & 30.52 & 26.31 & 29.75 & 25.29 & 7.14 & 28.09 & 41.16 & 33.33 \\
\hline DY & 18.28 & 29.61 & 22.77 & 29.11 & 16.20 & 24.81 & 13.45 & 23.49 & 18.18 & 27.21 & 36.51 & 29.66 & 16.67 & 25.19 \\
\hline SL & 25.81 & 35.18 & 37.22 & 34.76 & 25.55 & 31.58 & 24.89 & 29.52 & 27.27 & 31.03 & 26.98 & 35.16 & 41.67 & 29.54 \\
\hline $\mathbf{A P}$ & 15.05 & 29.61 & 22.22 & 28.56 & 14.64 & 22.51 & 20.08 & 27.19 & 23.69 & 29.32 & 30.15 & 33.39 & 33.33 & 17.81 \\
\hline $\mathrm{CO}$ & 8.60 & 21.02 & 3.88 & 16.34 & 3.42 & 14.43 & 8.63 & 22.73 & 7.71 & 22.26 & 28.57 & 25.45 & 12.5 & 35.35 \\
\hline DI & 2.15 & 8.32 & 3.33 & 14.65 & 2.18 & 9.46 & 3.61 & 12.73 & 5.78 & 18.59 & 0.79 & 5.14 & 0 & 0 \\
\hline FI & 87.10 & 25.35 & 82.22 & 30.35 & 81.62 & 31.13 & 81.32 & 30.14 & 79.61 & 29.61 & 84.12 & 27.78 & 91.67 & 15.43 \\
\hline
\end{tabular}

QL2 global health status/QoL, PF2 physical functioning, RF2 role functioning, EF emotional functioning, CF cognitive functioning, SF social functioning, FA fatigue, NV nausea vomiting, PA pain, DY dyspnea, SL sleeping difficulties/insomnia, AP appetite loss, CO constipation, DI di arrhea, FI financial difficulties. 
QoL Cancer Patients

Table 4. Means (SD) for EORTC QLQ-C30 by gender, marital status, household head, and educational attainment

\begin{tabular}{|c|c|c|c|c|c|c|c|c|c|c|c|c|c|}
\hline \multirow{2}{*}{ Scale } & \multicolumn{2}{|c|}{ Gender } & \multicolumn{4}{|c|}{ Marital status } & \multicolumn{2}{|c|}{ Household head } & \multicolumn{5}{|c|}{ Educational attainment } \\
\hline & Male & Female & Single & Married & Widow & Separated & Yes & No & None & Elem & HS & Vocational & College \\
\hline QL2 & $\begin{array}{l}54.41 \\
(20.81)\end{array}$ & $\begin{array}{l}56.71 \\
(20.26)\end{array}$ & $\begin{array}{l}54.41 \\
(20.81)\end{array}$ & $\begin{array}{l}56.71 \\
(20.26)\end{array}$ & $\begin{array}{l}55.69 \\
(20.49)\end{array}$ & $\begin{array}{l}52.08 \\
(18.63)\end{array}$ & $\begin{array}{l}55.13 \\
(19.85)\end{array}$ & $\begin{array}{l}56.08 \\
(21.21)\end{array}$ & $\begin{array}{l}55.95 \\
(17.15)\end{array}$ & $\begin{array}{l}52.83 \\
(22.84)\end{array}$ & $\begin{array}{l}54.98 \\
(20.15)\end{array}$ & $\begin{array}{l}55.79 \\
(22.62)\end{array}$ & $\begin{array}{l}59.03 \\
(17.87)\end{array}$ \\
\hline PF2 & $\begin{array}{l}76.39 \\
(24.54)\end{array}$ & $\begin{array}{l}77.86 \\
(23.75)\end{array}$ & $\begin{array}{l}76.39 \\
(24.54)\end{array}$ & $\begin{array}{l}77.86 \\
(23.75)\end{array}$ & $\begin{array}{l}71.88 \\
(24.18)\end{array}$ & $\begin{array}{l}75.42 \\
(24.43)\end{array}$ & $\begin{array}{l}78.57 \\
(22.52)\end{array}$ & $\begin{array}{l}75.78 \\
(25.57)\end{array}$ & $\begin{array}{l}75.24 \\
(21.33)\end{array}$ & $\begin{array}{l}73.53 \\
(26.18)\end{array}$ & $\begin{array}{l}77.32 \\
(23.13)\end{array}$ & $\begin{array}{l}72.75 \\
(30.94)\end{array}$ & $\begin{array}{l}81.86 \\
(20.33)\end{array}$ \\
\hline RF2 & $\begin{array}{l}65.11 \\
(33.49)\end{array}$ & $\begin{array}{l}68.71 \\
(31.91)\end{array}$ & $\begin{array}{l}65.11 \\
(33.49)\end{array}$ & $\begin{array}{l}68.71 \\
(31.91)\end{array}$ & $\begin{array}{l}63.05 \\
(32.63)\end{array}$ & $\begin{array}{l}66.67 \\
(33.33)\end{array}$ & $\begin{array}{l}67.55 \\
(32.23)\end{array}$ & $\begin{array}{l}66.42 \\
(33.21)\end{array}$ & $\begin{array}{l}69.05 \\
(24.40)\end{array}$ & $\begin{array}{l}62.84 \\
(34.67)\end{array}$ & $\begin{array}{l}70.01 \\
(29.80)\end{array}$ & $\begin{array}{l}57.61 \\
(36.12)\end{array}$ & $\begin{array}{l}69.46 \\
(33.39)\end{array}$ \\
\hline EF & $\begin{array}{l}71.98 \\
(22.08)\end{array}$ & $\begin{array}{l}69.24 \\
(21.29)\end{array}$ & $\begin{array}{l}71.98 \\
(22.08)\end{array}$ & $\begin{array}{l}69.24 \\
(21.29)\end{array}$ & $\begin{array}{l}69.31 \\
(21.57)\end{array}$ & $\begin{array}{l}71.35 \\
(19.47)\end{array}$ & $\begin{array}{l}72.13 \\
(21.17)\end{array}$ & $\begin{array}{l}69.01 \\
(22.14)\end{array}$ & $\begin{array}{l}75.00 \\
(10.76)\end{array}$ & $\begin{array}{l}67.28 \\
(24.19)\end{array}$ & $\begin{array}{l}71.20 \\
(20.65)\end{array}$ & $\begin{array}{l}73.55 \\
(19.82)\end{array}$ & $\begin{array}{l}71.56 \\
(21.53)\end{array}$ \\
\hline $\mathrm{CF}$ & $\begin{array}{l}86.84 \\
(18.00)\end{array}$ & $\begin{array}{l}84.47 \\
(19.92)\end{array}$ & $\begin{array}{l}86.83 \\
(18.00)\end{array}$ & $\begin{array}{l}84.47 \\
(19.92)\end{array}$ & $\begin{array}{l}83.33 \\
(17.08)\end{array}$ & $\begin{array}{l}89.58 \\
(17.08)\end{array}$ & $\begin{array}{l}86.60 \\
(17.59)\end{array}$ & $\begin{array}{l}84.63 \\
(20.36)\end{array}$ & $\begin{array}{l}85.71 \\
(11.50)\end{array}$ & $\begin{array}{l}82.46 \\
(20.39)\end{array}$ & $\begin{array}{l}87.66 \\
(17.71)\end{array}$ & $\begin{array}{l}84.42 \\
(22.33)\end{array}$ & $\begin{array}{l}86.01 \\
(18.52)\end{array}$ \\
\hline SF & $\begin{array}{l}70.29 \\
(32.23)\end{array}$ & $\begin{array}{l}79.19 \\
(25.96)\end{array}$ & $\begin{array}{l}70.29 \\
(32.23)\end{array}$ & $\begin{array}{l}79.19 \\
(25.95)\end{array}$ & $\begin{array}{l}77.77 \\
(27.89)\end{array}$ & $\begin{array}{l}78.12 \\
(26.33)\end{array}$ & $\begin{array}{l}73.39 \\
(31.11)\end{array}$ & $\begin{array}{l}76.42 \\
(27.71)\end{array}$ & $\begin{array}{l}95.24 \\
(12.59)\end{array}$ & $\begin{array}{l}72.96 \\
(30.12)\end{array}$ & $\begin{array}{l}76.87 \\
(27.54)\end{array}$ & $\begin{array}{l}75.36 \\
(32.15)\end{array}$ & $\begin{array}{l}72.84 \\
(30.87)\end{array}$ \\
\hline FA & $\begin{array}{l}23.99 \\
(1.53)\end{array}$ & $\begin{array}{l}20.54 \\
(1.33)\end{array}$ & $\begin{array}{l}23.99 \\
(24.59)\end{array}$ & $\begin{array}{l}20.54 \\
(22.34)\end{array}$ & $\begin{array}{l}27.03 \\
(26.07)\end{array}$ & $\begin{array}{l}22.92 \\
(16.96)\end{array}$ & $\begin{array}{l}22.01 \\
(23.28)\end{array}$ & $\begin{array}{l}22.39 \\
(23.74)\end{array}$ & $\begin{array}{l}22.22 \\
(15.71)\end{array}$ & $\begin{array}{l}25.10 \\
(26.11)\end{array}$ & $\begin{array}{l}22.55 \\
(22.55)\end{array}$ & $\begin{array}{l}23.91 \\
(24.56)\end{array}$ & $\begin{array}{l}18.41 \\
(21.91)\end{array}$ \\
\hline NV & $\begin{array}{l}4.60 \\
(11.94)\end{array}$ & $\begin{array}{l}6.77 \\
(15.67)\end{array}$ & $\begin{array}{l}4.60 \\
(11.93)\end{array}$ & $\begin{array}{l}6.77 \\
(15.67)\end{array}$ & $\begin{array}{l}5.83 \\
(13.66)\end{array}$ & $\begin{array}{l}2.08 \\
(5.69)\end{array}$ & $\begin{array}{l}4.59 \\
(12.01)\end{array}$ & $\begin{array}{l}6.85 \\
(15.72)\end{array}$ & $\begin{array}{l}2.38 \\
(6.29)\end{array}$ & $\begin{array}{l}7.65 \\
(17.72)\end{array}$ & $\begin{array}{l}5.39 \\
(11.49)\end{array}$ & $\begin{array}{l}3.98 \\
(15.78)\end{array}$ & $\begin{array}{l}5.13 \\
(13.01)\end{array}$ \\
\hline PA & $\begin{array}{l}33.20 \\
(28.14)\end{array}$ & $\begin{array}{l}30.33 \\
(27.22)\end{array}$ & $\begin{array}{l}33.20 \\
(28.14)\end{array}$ & $\begin{array}{l}30.33 \\
(27.23)\end{array}$ & $\begin{array}{l}34.44 \\
(28.43)\end{array}$ & $\begin{array}{l}28.12 \\
(24.13)\end{array}$ & $\begin{array}{l}32.39 \\
(26.87)\end{array}$ & $\begin{array}{l}31.05 \\
(28.49)\end{array}$ & $\begin{array}{l}28.57 \\
(15.85)\end{array}$ & $\begin{array}{l}33.45 \\
(27.51)\end{array}$ & $\begin{array}{l}33.49 \\
(28.75)\end{array}$ & $\begin{array}{l}31.16 \\
(28.02)\end{array}$ & $\begin{array}{l}27.85 \\
(26.52)\end{array}$ \\
\hline DY & $\begin{array}{l}20.10 \\
(28.22)\end{array}$ & $\begin{array}{l}15.10 \\
(24.27)\end{array}$ & $\begin{array}{l}20.10 \\
(28.22)\end{array}$ & $\begin{array}{l}15.11 \\
(24.27)\end{array}$ & $\begin{array}{l}23.33 \\
(27.65)\end{array}$ & $\begin{array}{l}20.83 \\
(29.50)\end{array}$ & $\begin{array}{l}16.60 \\
(25.31)\end{array}$ & $\begin{array}{l}18.39 \\
(27.33)\end{array}$ & $\begin{array}{l}9.52 \\
(16.26)\end{array}$ & $\begin{array}{l}20.49 \\
(29.08)\end{array}$ & $\begin{array}{l}17.48 \\
(25.72)\end{array}$ & $\begin{array}{l}21.74 \\
(29.16)\end{array}$ & $\begin{array}{l}13.75 \\
(23.51)\end{array}$ \\
\hline SL & $\begin{array}{l}29.96 \\
(33.29)\end{array}$ & $\begin{array}{l}25.54 \\
(30.22)\end{array}$ & $\begin{array}{l}29.96 \\
(33.29)\end{array}$ & $\begin{array}{l}25.54 \\
(30.23)\end{array}$ & $\begin{array}{l}31.11 \\
(34.09)\end{array}$ & $\begin{array}{l}31.25 \\
(30.95)\end{array}$ & $\begin{array}{l}27.79 \\
(32.09)\end{array}$ & $\begin{array}{l}27.53 \\
(31.54)\end{array}$ & $\begin{array}{l}14.28 \\
(26.22)\end{array}$ & $\begin{array}{l}30.37 \\
(32.69)\end{array}$ & $\begin{array}{l}28.10 \\
(31.12)\end{array}$ & $\begin{array}{l}28.98 \\
(34.86)\end{array}$ & $\begin{array}{l}24.71 \\
(31.08)\end{array}$ \\
\hline AP & $\begin{array}{l}19.84 \\
(27.47)\end{array}$ & $\begin{array}{l}21.34 \\
(28.04)\end{array}$ & $\begin{array}{l}19.84 \\
(27.47)\end{array}$ & $\begin{array}{l}21.34 \\
(28.04)\end{array}$ & $\begin{array}{l}27.78 \\
(30.17)\end{array}$ & $\begin{array}{l}14.58 \\
(17.07)\end{array}$ & $\begin{array}{l}19.62 \\
(26.28)\end{array}$ & $\begin{array}{l}21.60 \\
(29.14)\end{array}$ & $\begin{array}{l}23.81 \\
(25.19)\end{array}$ & $\begin{array}{l}25.67 \\
(31.26)\end{array}$ & $\begin{array}{l}20.75 \\
(26.25)\end{array}$ & $\begin{array}{l}18.84 \\
(23.98)\end{array}$ & $\begin{array}{l}16.08 \\
(27.08)\end{array}$ \\
\hline $\mathrm{CO}$ & $\begin{array}{l}7.26 \\
(21.23)\end{array}$ & $\begin{array}{l}7.19 \\
(20.91)\end{array}$ & $\begin{array}{l}7.26 \\
(21.22)\end{array}$ & $\begin{array}{l}7.19 \\
(20.91)\end{array}$ & $\begin{array}{l}12.22 \\
(29.41)\end{array}$ & $\begin{array}{l}12.50 \\
(34.16)\end{array}$ & $\begin{array}{l}7.29 \\
(21.45)\end{array}$ & $\begin{array}{l}7.17 \\
(20.68)\end{array}$ & $0(0)$ & $\begin{array}{l}6.42 \\
(18.89)\end{array}$ & $\begin{array}{l}7.35 \\
(20.80)\end{array}$ & $\begin{array}{l}8.69 \\
(21.58)\end{array}$ & $\begin{array}{l}7.69 \\
(23.63)\end{array}$ \\
\hline DI & $\begin{array}{l}3.76 \\
(14.09)\end{array}$ & $\begin{array}{l}3.11 \\
(12.59)\end{array}$ & $\begin{array}{l}3.76 \\
(14.08)\end{array}$ & $\begin{array}{l}3.11 \\
(12.59)\end{array}$ & $\begin{array}{l}1.67 \\
(7.32)\end{array}$ & $\begin{array}{l}4.16 \\
(16.67)\end{array}$ & $\begin{array}{l}4.53 \\
(15.22)\end{array}$ & $\begin{array}{l}2.34 \\
(11.06)\end{array}$ & $\begin{array}{l}9.52 \\
(25.19)\end{array}$ & $\begin{array}{l}2.47 \\
(8.76)\end{array}$ & $\begin{array}{l}4.25 \\
(15.63)\end{array}$ & $\begin{array}{l}5.79 \\
(16.18)\end{array}$ & $\begin{array}{l}2.09 \\
(11.34)\end{array}$ \\
\hline FI & $\begin{array}{l}83.39 \\
(28.12)\end{array}$ & $\begin{array}{l}80.33 \\
(30.85)\end{array}$ & $\begin{array}{l}83.39 \\
(28.12)\end{array}$ & $\begin{array}{l}80.34 \\
(30.85)\end{array}$ & $\begin{array}{l}75.56 \\
(32.97)\end{array}$ & $\begin{array}{l}85.42 \\
(27.13)\end{array}$ & $\begin{array}{l}80.88 \\
(29.93)\end{array}$ & $\begin{array}{l}82.71 \\
(29.27)\end{array}$ & $\begin{array}{l}85.71 \\
(26.23)\end{array}$ & $\begin{array}{l}82.71 \\
(28.46)\end{array}$ & $\begin{array}{l}80.88 \\
(30.67)\end{array}$ & $\begin{array}{l}81.16 \\
(30.35)\end{array}$ & $\begin{array}{l}82.29 \\
(29.29)\end{array}$ \\
\hline
\end{tabular}

QL2 global health status/QoL, PF2 physical functioning, RF2 role functioning, EF emotional functioning, CF cognitive functioning, SF social functioning, FA fatigue, NV nausea vomiting, PA pain, DY dyspnea, SL sleeping difficulties/insomnia, AP appetite loss, CO constipation, DI diarrhea, FI financial difficulties.

Table 5. Means (SD) for EORTC QLQ-C30 by presence of insurance and Philippine income bracket

\begin{tabular}{|c|c|c|c|c|c|c|c|c|c|c|c|}
\hline \multirow{2}{*}{ Scale } & \multicolumn{2}{|c|}{ Insurance Income } & \multicolumn{9}{|c|}{ Bracket } \\
\hline & Present & Absent & 1 & 2 & 3 & 4 & 5 & 6 & 7 & 8 & 9 \\
\hline QL2 & $52.78(22.40)$ & $55.83(20.39)$ & 55.95 (21.53) & $55.97(20.53)$ & $54.43(20.58)$ & 56.09 (19.17) & $54.65(20.84)$ & 55.55 (17.14) & $46.08(23.95)$ & $65.48(22.28)$ & 59.93 (19.43) \\
\hline PF2 & 70.59 (28.14) & 77.67 (23.73) & 79.76 (22.83) & 77.71 (23.17) & 73.54 (25.95) & $79.10(21.58)$ & $74.31(27.76)$ & $77.22(23.50)$ & 70.19 (30.19) & $72.38(24.77)$ & $80.51(25.98)$ \\
\hline RF2 & 61.97 (37.45) & $67.37(32.31)$ & 70.09 (31.49) & $69.07(32.40)$ & 62.85 (33.02) & $69.65(30.56)$ & $63.23(35.48)$ & $61.11(33.93)$ & $55.88(33.30)$ & $57.14(40.66)$ & $69.87(36.52)$ \\
\hline EF & $69.23(25.58)$ & 70.67 (21.39) & 69.57 (20.46) & 72.58 (19.34) & $70.31(23.38)$ & $67.78(23.61)$ & 70.34 (20.84) & 70.48 (15.54) & $66.17(32.07)$ & $79.76(26.72)$ & $71.79(26.04)$ \\
\hline CF & $84.18(19.09)$ & $85.72(19.05)$ & 84.82 (21.17) & 84.10 (18.73) & $86.97(17.63)$ & $86.82(15.76)$ & $84.80(24.05)$ & $90.28(11.95)$ & $83.33(24.29)$ & $95.24(8.13)$ & $85.25(20.72)$ \\
\hline SF & $66.24(34.09)$ & $75.60(28.98)$ & $75.15(27.58)$ & $75.00(27.66)$ & 71.01 (32.46) & $81.09(26.57)$ & 76.47 (29.33) & $76.39(27.33)$ & $63.73(41.34)$ & $90.47(16.26)$ & 71.79 (36.74) \\
\hline FA & $31.91(30.92)$ & $21.43(22.67)$ & $20.73(21.79)$ & $22.07(23.95)$ & $24.19(24.50)$ & $24.71(20.91)$ & $22.55(26.02)$ & $18.06(18.77)$ & $32.67(30.55)$ & $14.28(17.81)$ & $14.10(25.83)$ \\
\hline NV & $7.26(20.87)$ & $5.61(13.37)$ & $6.39(14.19)$ & $6.79(14.77)$ & $6.25(15.15)$ & $3.48(12.15)$ & $8.33(19.35)$ & $2.08(7.47)$ & $0.98(4.04)$ & 4.76 (12.59) & $3.84(8.57)$ \\
\hline PA & 40.59 (32.17) & $31.01(27.21)$ & $32.87(27.80)$ & 33.22 (29.43) & 32.29 (26.58) & $28.11(21.75)$ & $29.41(27.84)$ & 34.03 (25.29) & 37.25 (35.12) & 38.09 (34.31) & $20.51(29.17)$ \\
\hline DY & $18.80(28.40)$ & 17.41 (26.19) & 13.69 (24.73) & $20.17(27.96)$ & 19.09 (27.67) & $20.39(25.92)$ & $13.73(23.38)$ & $16.67(19.66)$ & $27.45(35.81)$ & $9.52(16.26)$ & $6.41(18.90)$ \\
\hline SL & 30.77 (35.36) & $27.42(31.51)$ & $30.95(34.86)$ & $30.92(32.12)$ & $21.87(30.52)$ & $26.86(26.74)$ & $29.41(34.58)$ & $19.44(25.85)$ & 35.29 (34.29) & $9.52(16.26)$ & $23.07(32.34)$ \\
\hline AP & $28.21(37.10)$ & $20.03(26.84)$ & $19.64(28.84)$ & $22.15(26.56)$ & $23.26(29.46)$ & 20.39 (25.92) & $19.61(29.72)$ & $18.05(25.96)$ & $21.57(28.72)$ & 4.76 (12.59) & $14.10(28.55)$ \\
\hline $\mathrm{CO}$ & $14.53(31.33)$ & $6.65(19.95)$ & $9.52(24.27)$ & $8.33(21.44)$ & 4.51 (15.76) & $6.46(22.65)$ & $4.90(16.68)$ & $1.38(6.80)$ & $9.80(28.29)$ & $19.05(26.23)$ & $6.41(23.13)$ \\
\hline DI & $5.12(14.38)$ & $3.29(13.25)$ & 4.76 (15.41) & $3.29(14.23)$ & $3.47(11.32)$ & $1.49(6.94)$ & $3.92(17.91)$ & $1.38(6.80)$ & 3.92 (16.17) & 4.76 (12.59) & $3.84(14.38)$ \\
\hline FI & $87.18(28.22)$ & 81.38 (29.67) & $81.25(29.94)$ & $82.24(28.94)$ & $84.72(26.89)$ & 84.07 (27.44) & 77.45 (33.56) & $70.83(33.06)$ & 80.39 (33.45) & 80.95 (37.79) & $82.05(34.29)$ \\
\hline
\end{tabular}

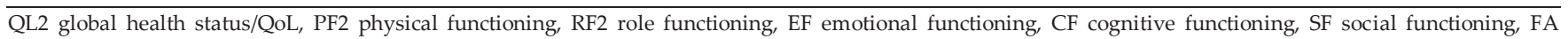
fatigue, NV nausea vomiting, PA pain, DY dyspnea, SL sleeping difficulties/insomnia, AP appetite loss, CO constipation, DI diarrhea, FI financial difficulties. 
QoL Cancer Patients

Table 6. Means for EQ5D by age category

\begin{tabular}{|c|c|c|c|c|c|c|c|c|c|}
\hline \multirow{2}{*}{$\begin{array}{l}\text { EQ-5D } \\
\text { DIMENSION }\end{array}$} & \multirow[b]{2}{*}{ Level } & \multicolumn{7}{|c|}{ Age Category (\%) } & \multirow{2}{*}{ TOTAI } \\
\hline & & $19-29 y$ & $30-39 y$ & $40-49 y$ & $50-59 y$ & $60-69 y$ & $70-79 y$ & $\geq 80 y$ & \\
\hline Mobility & 1 & 74.19 & 75.00 & 77.57 & 69.88 & 65.29 & 42.86 & 87.50 & 69.35 \\
\hline \multirow[t]{2}{*}{$(\mathrm{p}=0.001)$} & 3 & 19.35 & 25.00 & 22.43 & 28.31 & 33.88 & 57.14 & 12.50 & 29.53 \\
\hline & 5 & 6.45 & 0.00 & 0.00 & 1.81 & 0.83 & 0.00 & 0.00 & 1.12 \\
\hline Personal Care & 1 & 74.19 & 85.00 & 80.37 & 77.71 & 79.34 & 69.05 & 87.50 & 78.69 \\
\hline \multirow[t]{2}{*}{$(\mathrm{p}=0.560)$} & 3 & 16.13 & 13.33 & 17.76 & 18.67 & 15.70 & 21.43 & 12.50 & 17.20 \\
\hline & 5 & 9.68 & 1.67 & 1.87 & 3.61 & 4.96 & 9.52 & 0.00 & 4.11 \\
\hline Usual Activity & 1 & 51.61 & 41.67 & 34.58 & 35.54 & 35.54 & 21.43 & 37.50 & 35.89 \\
\hline \multirow{2}{*}{$(p=0.077)$} & 3 & 29.03 & 41.67 & 49.53 & 51.20 & 47.11 & 52.38 & 12.50 & 47.10 \\
\hline & 5 & 19.35 & 16.67 & 15.89 & 13.25 & 17.36 & 26.19 & 50.00 & 17.01 \\
\hline Pain & 1 & 45.16 & 21.67 & 27.10 & 24.70 & 22.31 & 23.81 & 25.00 & 25.42 \\
\hline \multirow{2}{*}{$(\mathrm{p}=0.362)$} & 3 & 45.16 & 61.67 & 63.55 & 65.66 & 69.42 & 64.29 & 50.00 & 64.11 \\
\hline & 5 & 9.68 & 16.67 & 9.35 & 9.64 & 8.26 & 11.90 & 25.00 & 10.47 \\
\hline \multirow{3}{*}{$\begin{array}{l}\text { Anxiety/Depression } \\
(\mathrm{p}=0.980)\end{array}$} & 1 & 25.03 & 43.33 & 37.38 & 37.95 & 38.02 & 40.48 & 50.00 & 38.32 \\
\hline & 3 & 61.29 & 48.33 & 57.01 & 56.02 & 54.55 & 54.76 & 50.00 & 55.14 \\
\hline & 5 & 9.68 & 8.33 & 5.61 & 6.02 & 7.44 & 4.76 & 0.00 & 6.54 \\
\hline
\end{tabular}

Table 7. Means for EQ5D by civil status

\begin{tabular}{|c|c|c|c|c|c|c|}
\hline \multirow[t]{2}{*}{ EQ-5D DIMENSION } & \multirow[b]{2}{*}{ Level } & \multicolumn{4}{|c|}{ Civil Status } & \multirow{2}{*}{ TOTAL } \\
\hline & & Single & Married & Widowed & Separated & \\
\hline \multirow{3}{*}{$\begin{array}{l}\text { Mobility } \\
(\mathrm{p}=0.016)\end{array}$} & 1 & 77.42 & 71.03 & 50.00 & 68.75 & 69.35 \\
\hline & 3 & 20.97 & 27.71 & 50.00 & 31.25 & 29.53 \\
\hline & 5 & 1.61 & 1.26 & 0.00 & 0.00 & 1.12 \\
\hline \multirow{3}{*}{$\begin{array}{l}\text { Personal Care } \\
(\mathrm{p}=0.945)\end{array}$} & 1 & 83.87 & 77.83 & 78.33 & 81.25 & 78.69 \\
\hline & 3 & 12.90 & 17.88 & 18.33 & 12.50 & 17.20 \\
\hline & 5 & 3.23 & 4.28 & 3.33 & 6.25 & 4.11 \\
\hline \multirow{3}{*}{$\begin{array}{l}\text { Usual Activity } \\
(\mathrm{p}=0.309)\end{array}$} & 1 & 48.39 & 33.50 & 36.67 & 43.75 & 35.89 \\
\hline & 3 & 38.71 & 48.87 & 48.33 & 31.25 & 47.10 \\
\hline & 5 & 12.90 & 17.63 & 15.00 & 25.00 & 17.01 \\
\hline \multirow{3}{*}{$\begin{array}{l}\text { Pain } \\
(p=0.180)\end{array}$} & 1 & 38.71 & 22.92 & 25.00 & 37.50 & 25.42 \\
\hline & 3 & 54.84 & 65.74 & 65.00 & 56.25 & 64.11 \\
\hline & 5 & 6.45 & 11.34 & 10.00 & 6.25 & 10.47 \\
\hline \multirow{3}{*}{$\begin{array}{l}\text { Anxiety/Depression } \\
(\mathrm{p}=0.508)\end{array}$} & 1 & 35.48 & 38.04 & 41.67 & 43.75 & 38.32 \\
\hline & 3 & 62.90 & 54.66 & 53.33 & 43.75 & 55.14 \\
\hline & 5 & 1.61 & 7.30 & 5.00 & 12.50 & 6.54 \\
\hline
\end{tabular}

Table 8. Means for EQ5D by income bracket

\begin{tabular}{|c|c|c|c|c|c|c|c|c|c|c|c|}
\hline \multirow{2}{*}{$\begin{array}{c}\text { EQ-5D } \\
\text { DIMENSION }\end{array}$} & \multirow{2}{*}{ Level } & \multicolumn{9}{|c|}{ Income Bracket } & \multirow{2}{*}{ TOTAL } \\
\hline & & 1 & 2 & 3 & 4 & 5 & 6 & 7 & 8 & 9 & \\
\hline \multirow{3}{*}{$\begin{array}{l}\text { Mobility } \\
(p=0.517)\end{array}$} & 1 & 67.86 & 65.13 & 64.58 & 76.12 & 79.41 & 75.00 & 58.82 & 85.71 & 84.62 & 69.35 \\
\hline & 3 & 31.25 & 32.89 & 35.42 & 22.39 & 17.65 & 25.00 & 41.18 & 14.29 & 15.38 & 29.53 \\
\hline & 5 & 0.89 & 1.97 & 0.00 & 1.49 & 2.94 & 0.00 & 0.00 & 0.00 & 0.00 & 1.12 \\
\hline \multirow{3}{*}{$\begin{array}{l}\text { Personal Care } \\
(\mathrm{p}=0.002)\end{array}$} & 1 & 82.14 & 80.26 & 77.08 & 86.57 & 70.59 & 58.33 & 64.71 & 71.43 & 80.77 & 78.69 \\
\hline & 3 & 14.29 & 14.47 & 19.79 & 11.94 & 29.41 & 41.67 & 23.53 & 0.00 & 11.54 & 17.20 \\
\hline & 5 & 3.57 & 5.26 & 3.13 & 1.49 & 0.00 & 0.00 & 11.76 & 28.57 & 7.69 & 4.11 \\
\hline \multirow{2}{*}{$\begin{array}{l}\text { Usual Activity } \\
(\mathrm{p}=0.191)\end{array}$} & 3 & 40.18 & 45.39 & 60.42 & 43.28 & 50.00 & 58.33 & 52.94 & 28.57 & 34.62 & 47.10 \\
\hline & 5 & 20.54 & 20.39 & 14.58 & 11.94 & 11.76 & 16.67 & 11.76 & 28.57 & 11.54 & 17.01 \\
\hline \multirow{3}{*}{$\begin{array}{l}\text { Pain } \\
(p=0.147)\end{array}$} & 1 & 28.57 & 21.05 & 20.83 & 34.33 & 20.59 & 33.33 & 11.76 & 0.00 & 46.15 & 25.42 \\
\hline & 3 & 62.50 & 66.45 & 64.58 & 59.70 & 70.59 & 62.50 & 82.35 & 85.71 & 42.31 & 64.11 \\
\hline & 5 & 8.93 & 12.50 & 14.58 & 5.97 & 8.82 & 4.17 & 5.88 & 14.29 & 11.54 & 10.47 \\
\hline \multirow{3}{*}{$\begin{array}{l}\text { Anxiety/Depression } \\
(p=0.770)\end{array}$} & 1 & 39.29 & 35.53 & 39.58 & 37.31 & 50.00 & 25.00 & 41.18 & 57.14 & 38.46 & 38.32 \\
\hline & 3 & 52.68 & 59.87 & 51.04 & 56.72 & 47.06 & 66.67 & 58.82 & 42.86 & 50.00 & 55.14 \\
\hline & 5 & 8.04 & 4.61 & 9.38 & 5.97 & 2.94 & 8.33 & 0.00 & 0.00 & 11.54 & 6.54 \\
\hline
\end{tabular}


Table 9. Means for EQ5D by type of work

\begin{tabular}{|c|c|c|c|c|c|c|c|c|c|c|c|c|c|}
\hline \multirow{2}{*}{$\begin{array}{l}\text { Q-5D } \\
\text { DIMENSION }\end{array}$} & \multirow{2}{*}{ Level } & \multicolumn{11}{|c|}{ Type of Work } & \multirow{2}{*}{ TOTAL } \\
\hline & & 1 & 2 & 3 & 4 & 5 & 6 & 7 & 8 & 10 & 11 & 12 & \\
\hline \multirow{3}{*}{$\begin{array}{l}\text { Mobility } \\
(p=0.934)\end{array}$} & 1 & 81.82 & 67.86 & 71.43 & 80.77 & 70.65 & 70.69 & 68.75 & 65.00 & 62.96 & 61.54 & 70.00 & 69.35 \\
\hline & 3 & 18.18 & 32.14 & 28.57 & 15.38 & 28.36 & 29.31 & 31.25 & 32.50 & 35.19 & 34.62 & 30.00 & 29.53 \\
\hline & 5 & 0.00 & 0.00 & 0.00 & 3.85 & 1.00 & 0.00 & 0.00 & 2.50 & 1.85 & 3.85 & 0.00 & 1.12 \\
\hline \multirow{3}{*}{$\begin{array}{l}\text { Personal Care } \\
(p=0.476)\end{array}$} & 1 & 81.82 & 75.00 & 71.43 & 84.62 & 80.10 & 87.93 & 71.88 & 70.00 & 75.93 & 80.77 & 80.00 & 78.69 \\
\hline & 3 & 18.18 & 21.43 & 23.81 & 7.69 & 15.92 & 8.62 & 21.88 & 30.00 & 14.81 & 19.23 & 20.00 & 17.20 \\
\hline & 5 & 0.00 & 3.57 & 4.76 & 7.69 & 3.98 & 3.45 & 6.25 & 0.00 & 9.26 & 0.00 & 0.00 & 4.11 \\
\hline \multirow{3}{*}{$\begin{array}{l}\text { Usual Activity } \\
(\mathrm{p}=0.170)\end{array}$} & 1 & 36.36 & 35.71 & 19.05 & 46.15 & 40.30 & 24.14 & 43.75 & 25.00 & 31.48 & 42.31 & 50.00 & 35.89 \\
\hline & 3 & 54.55 & 50.00 & 71.43 & 30.77 & 44.78 & 55.17 & 46.88 & 60.00 & 40.74 & 34.62 & 30.00 & 47.10 \\
\hline & 5 & 9.00 & 14.29 & 9.52 & 23.08 & 14.93 & 20.69 & 9.38 & 15.00 & 27.78 & 23.08 & 20.00 & 17.01 \\
\hline \multirow{3}{*}{$\begin{array}{l}\text { Pain } \\
(p=0.474)\end{array}$} & 1 & 27.27 & 25.00 & 9.52 & 34.62 & 25.37 & 13.79 & 28.13 & 22.50 & 38.89 & 26.92 & 30.00 & 25.42 \\
\hline & 3 & 72.73 & 64.29 & 85.71 & 57.69 & 61.19 & 74.14 & 65.63 & 70.00 & 51.85 & 65.38 & 60.00 & 64.11 \\
\hline & 5 & 0.00 & 10.71 & 4.76 & 7.69 & 13.43 & 12.07 & 6.25 & 7.50 & 9.26 & 7.69 & 10.00 & 10.47 \\
\hline \multirow{3}{*}{$\begin{array}{l}\text { Anxiety/Depressi } \\
\text { on } \\
(\mathrm{p}=0.582)\end{array}$} & 1 & 27.27 & 35.71 & 42.86 & 26.92 & 41.29 & 43.10 & 28.13 & 27.50 & 42.59 & 46.15 & 30.00 & 38.32 \\
\hline & 3 & 72.73 & 58.93 & 42.86 & 69.23 & 53.23 & 53.45 & 62.50 & 60.00 & 50.00 & 42.31 & 70.00 & 55.14 \\
\hline & 5 & 0.00 & 5.36 & 14.29 & 3.85 & 5.47 & 3.45 & 9.38 & 12.50 & 7.41 & 11.54 & 0.00 & 6.54 \\
\hline
\end{tabular}

Table 10. Means for EQ5D by educational attainment

\begin{tabular}{|c|c|c|c|c|c|c|c|}
\hline \multirow{2}{*}{ EQ-5D DIMENSION } & \multirow{2}{*}{ Level } & \multicolumn{5}{|c|}{ Educational Attainment } & \multirow{2}{*}{ TOTAL } \\
\hline & & None & Elementary & High School & Vocational & College & \\
\hline Mobility & 1 & 71.43 & 65.93 & 67.65 & 73.91 & 73.43 & 69.35 \\
\hline \multirow[t]{2}{*}{$(p=0.645)$} & 3 & 28.57 & 33.33 & 30.39 & 23.91 & 26.53 & 29.53 \\
\hline & 5 & 0.00 & 0.74 & 1.96 & 2.17 & 0.00 & 1.12 \\
\hline \multirow{3}{*}{$\begin{array}{l}\text { Personal Care } \\
(\mathrm{p}=0.763)\end{array}$} & 1 & 71.43 & 77.78 & 76.47 & 78.26 & 83.22 & 78.69 \\
\hline & 3 & 28.57 & 16.30 & 19.12 & 17.39 & 14.69 & 17.20 \\
\hline & 5 & 0.00 & 5.93 & 4.41 & 4.35 & 2.10 & 4.11 \\
\hline \multirow{3}{*}{$\begin{array}{l}\text { Usual Activity } \\
(\mathrm{p}=0.201)\end{array}$} & 1 & 42.86 & 28.89 & 37.25 & 43.48 & 37.76 & 35.89 \\
\hline & 3 & 57.14 & 46.67 & 46.57 & 41.30 & 49.65 & 47.10 \\
\hline & 5 & 0.00 & 24.44 & 16.18 & 15.22 & 12.59 & 17.01 \\
\hline \multirow{3}{*}{$\begin{array}{l}\text { Pain } \\
(p=0.800)\end{array}$} & 1 & 28.57 & 21.48 & 24.51 & 26.09 & 30.07 & 25.42 \\
\hline & 3 & 71.43 & 65.93 & 64.22 & 65.22 & 61.54 & 64.11 \\
\hline & 5 & 0.00 & 12.59 & 11.27 & 8.70 & 8.39 & 10.47 \\
\hline \multirow{3}{*}{$\begin{array}{l}\text { Anxiety/Depression } \\
(p=0.729)\end{array}$} & 1 & 14.29 & 34.81 & 38.24 & 41.30 & 41.96 & 38.32 \\
\hline & 3 & 85.71 & 59.26 & 53.92 & 52.17 & 52.45 & 55.14 \\
\hline & 5 & 0.00 & 5.93 & 7.84 & 6.52 & 5.59 & 6.54 \\
\hline
\end{tabular}

Table 11. Frequency for EQ-5D QOL by cancer site

\begin{tabular}{|c|c|c|c|c|c|c|c|c|c|c|c|c|c|c|c|c|}
\hline $\begin{array}{c}\text { EQ-5D } \\
\text { DIMENSION }\end{array}$ & Level & \multicolumn{14}{|c|}{ Cancer Site } & Total \\
\hline \multirow{3}{*}{$\begin{array}{l}\text { Mobility } \\
(\mathrm{p}=0.013)\end{array}$} & 1 & 72 & 3 & 3 & 95 & 3 & 2 & 24 & 4 & 84 & 1 & 2 & 1 & 29 & 48 & 371 \\
\hline & 3 & 15 & 1 & 1 & 55 & 1 & 0 & 29 & 1 & 17 & 0 & 3 & 3 & 13 & 19 & 158 \\
\hline & 5 & 2 & 0 & 0 & 1 & 0 & 0 & 0 & 0 & 1 & 0 & 0 & 0 & 1 & 1 & 6 \\
\hline \multirow{3}{*}{$\begin{array}{l}\text { Personal Care } \\
(\mathrm{p}=0.550)\end{array}$} & 1 & 72 & 4 & 4 & 114 & 3 & 2 & 36 & 4 & 88 & 1 & 4 & 1 & 33 & 55 & 421 \\
\hline & 3 & 15 & 0 & 0 & 30 & 1 & 0 & 14 & 1 & 12 & 0 & 1 & 2 & 6 & 10 & 92 \\
\hline & 5 & 2 & 0 & 0 & 7 & 0 & 0 & 3 & 0 & 2 & 0 & 0 & 1 & 4 & 3 & 22 \\
\hline \multirow{3}{*}{$\begin{array}{l}\text { Pain } \\
(\mathrm{p}=0.015)\end{array}$} & 1 & 14 & 1 & 1 & 38 & 2 & 1 & 10 & 2 & 32 & 1 & 0 & 0 & 13 & 21 & 136 \\
\hline & 3 & 62 & 3 & 3 & 105 & 1 & 1 & 35 & 3 & 64 & 0 & 5 & 2 & 20 & 39 & 343 \\
\hline & 5 & 13 & 0 & 0 & 8 & 1 & 0 & 8 & 0 & 6 & 0 & 0 & 3 & 10 & 8 & 56 \\
\hline \multirow{3}{*}{$\begin{array}{l}\text { Anxiety/ } \\
\text { Depression } \\
(\mathrm{p}=0.438)\end{array}$} & 1 & 31 & 3 & 3 & 54 & 1 & 0 & 23 & 3 & 40 & 1 & 3 & 0 & 14 & 29 & 205 \\
\hline & 3 & 56 & 1 & 1 & 88 & 2 & 2 & 26 & 2 & 54 & 0 & 2 & 3 & 23 & 35 & 295 \\
\hline & 5 & 2 & 0 & 0 & 9 & 1 & 0 & 4 & 0 & 8 & 0 & 0 & 1 & 6 & 4 & 35 \\
\hline
\end{tabular}


The effect of cancer site and stage

Bladder cancer was associated with poorer QoL as compared with all other histologies (Table 11). This particular histology was also associated with higher fatigue (vs. colorectal/breast/melanoma). Breast cancer patients tended to have less pain than bladder cancer patients. As expected, lung cancer patients were associated with more dyspnea. Surprisingly, bladder cancer was also associated with increase in dyspnea (vs. colorectal/breast/lymphoma). Bladder cancer was also associated with less appetite (vs. colorectal/head and neck/esophagus/lung/breast/prostate). The rest of the associations did not reach statistical significance.

Decrease in mobility $(p=0.005)$, decrease in usual activities $(p=0.015)$, and decrease in personal care $(p=0.002)$ were associated with increase in cancer stage according to EQ-5D (Table 12). The rest of the associations did not reach statistical significance.

Table 12. Frequency for EQ-5D QOL by cancer stage

\begin{tabular}{|c|c|c|c|c|c|c|}
\hline \multirow{2}{*}{$\begin{array}{l}\text { EQ-5D } \\
\text { DIMENSION }\end{array}$} & \multirow{2}{*}{ Level } & \multicolumn{4}{|c|}{ Cancer Stage } & \multirow{2}{*}{ Total } \\
\hline & & 1 & 2 & 3 & 4 & \\
\hline \multirow{3}{*}{$\begin{array}{l}\text { Mobility } \\
(p=0.005)\end{array}$} & 1 & 27 & 98 & 130 & 116 & 371 \\
\hline & 3 & 6 & 22 & 61 & 69 & 158 \\
\hline & 5 & 0 & 0 & 4 & 2 & 6 \\
\hline \multirow{3}{*}{$\begin{array}{l}\text { Personal Care } \\
(p=0.002)\end{array}$} & 1 & 30 & 106 & 155 & 130 & 421 \\
\hline & 3 & 2 & 13 & 30 & 47 & 92 \\
\hline & 5 & 1 & 1 & 10 & 10 & 22 \\
\hline \multirow{3}{*}{$\begin{array}{l}\text { Usual Activity } \\
(\mathrm{p}=0.015)\end{array}$} & 1 & 16 & 50 & 75 & 51 & 192 \\
\hline & 3 & 15 & 55 & 90 & 92 & 252 \\
\hline & 5 & 2 & 15 & 30 & 44 & 91 \\
\hline \multirow{3}{*}{$\begin{array}{l}\text { Pain } \\
(p=0.059)\end{array}$} & 1 & 11 & 36 & 50 & 39 & 136 \\
\hline & 3 & 22 & 76 & 125 & 120 & 343 \\
\hline & 5 & 0 & 8 & 20 & 28 & 56 \\
\hline \multirow{3}{*}{$\begin{array}{l}\text { Anxiety/Depression } \\
(p=0.254)\end{array}$} & 1 & 17 & 48 & 70 & 70 & 205 \\
\hline & 3 & 15 & 67 & 106 & 107 & 295 \\
\hline & 5 & 1 & 5 & 19 & 10 & 35 \\
\hline
\end{tabular}

\section{Discussion}

In this study, two types of quality of life (general quality of life and cancer-specific quality of life) were quantified and correlated with the demographics of the usual Filipino cancer patient seen in the charity outpatient clinics. Newlydiagnosed cancer patients were recruited in this study because we wanted to know who will need additional care or referrals during treatment. Younger patients had better physical and cognitive function while older patients tended to perform worse in terms of role functioning and emotional functioning. This could be explained by the younger people having abler bodies and less comorbidities at baseline while as age increased, comorbidities and age-related degenerative changes also increased. Because of this, younger patients also tended to withstand the side effects of chemotherapy or radiotherapy that they might need in the future. Older people were also more dependent because of these and this could affect their role functioning and emotional functioning. They might be more neglected than their younger counterparts and this might lead to undetected mood problems. In fact, in a study done by Botega, ${ }^{12}$ undetected psychiatric disorders, in admitted cancer patients, especially in the elderly population, reached $30 \%$. It might be warranted to spend more time talking to older cancer patients to detect these disorders or to refer them to Psychiatry early in their treatment course. Our results are the opposite of a study among breast cancer patients in a Swedish population ${ }^{13}$ and a UK population ${ }^{14}$ wherein the older population had more positive emotional functioning. This might be due to the government-based health insurance that was given to them.

The patients who belonged to the lower income bracket scored higher in personal care questions. This could be because they were conditioned early on to tend to their needs and not be dependent on someone else. For their more affluent counterparts, who lived with helpers at home, personal care could be dependent on someone else. These people and their families might need extra counseling during the course of the disease and treatment so that helplessness would not be experienced much by these patients. Empowering these patients early on might be warranted. However, in a study by Zigmond, ${ }^{15}$ loss of employment temporarily upon cancer diagnosis was associated with lower social functioning and job discrimination. In a study by Hermann, ${ }^{16}$ low income patients had lower physical functioning and emotional functioning.

Surprisingly, educational status, presence of insurance, type of work, and income did not significantly alter the quality of life of patients at the onset of their cancer treatment. The effect might be more pronounced as the patients move on to chemotherapy, surgery, and other forms of treatment that would need substantial financial support. The financial catastrophe brought about by cancer might be seen in a longitudinal study that could also measure the changes in QoL.

At start of cancer treatment or upon diagnosis, what seemed to be more important was the amount of social support that the patient felt he/she has. The patient could be thinking of support from friends or family in terms of emotion or financial. Nonetheless, knowing that someone was there for you throughout the course of treatment could lead to better QoL.

The associations that we established for cancer stage and QoL were expected. Patient QoL was better for early stage cancers. For histology-specific QoL, bladder cancer patients seemed to perform the poorest. This histology was associated with decrease in physical functioning, increase in fatigue, increase in pain, increase in dyspnea, and decrease in appetite. These patients might need to be given early maximal treatment and be referred early on to other relevant sub-specialities (psychiatry, hospice, pain, gastroenterology). 


\section{Limitations}

The major limitation of our study was that we concentrated on recruiting charity cancer patients because of their proximity to our own clinics and their numbers. The above average Filipino patient when it came to income bracket was admittedly under-represented.

\section{Summary}

This is a baseline study on self-reported QoL among newly-diagnosed cancer patients in the medical oncology clinics of the Cancer Institute of the University of the Philippines-Philippine General Hospital. Here so showed that the physical burden of cancer was better tolerated by younger patients. Older patients tended to perform poorer in terms of role and emotional functioning that might make them more susceptible to mood disorders further in the course of their disease. Early referrals to other subspecialties might be warranted for these patient subsets. Finances and work-related factors did not seem to alter QoL early in the course of the patient's disease, while having advanced disease upon diagnosis was associated with poor QoL. However, having some close friends increased the QoL of our newly-diagnosed cancer patients. A longitudinal study which followed-up these patients to know the impact of finances, stage, histology, and other aspects of cancer is now being analyzed to further understand the QoL of cancer patients.

\section{References}

1. Snaith RP. The Hospital Anxiety and Depression Scale. Health Quality Life Outcomes. 2003; 1:29.

2. Slevin ML, Plant H, Lynch D, Drinkwater J, Gregory WM. Who should measure quality of life, the doctor or the patient? Br J Cancer. 1988; 57(1):109-12.

3. Bowling A. Measuring Health: A Review of Quality of Life Measurement Scales. Milton Keynes: Open University Press, 1991.

4. McDowell I, Newell C. Measuring Health. A Guide to Rating Scales and Questionnaires. New York: Oxford University Press, 1987.

5. Jaeschke R, Guyatt G. How to develop and validate a new quality of life instrument. In: Snilker B, ed. Quality of Life Assessment in Clinical Trials. New York: Raven Press; 1990. pp. 47-57.

6. Dancey J, Zee B, Osoba D, Whitehead M, et al. Quality of life scores: an independent prognostic variable in a general population of cancer patients receiving chemotherapy. The National Cancer Institute of Canada Clinical Trials Group. Qual Life Res. 1997; 6(2):151-8.

7. Wang YB, Chen MH, Yan K, Yang W, Dai Y, Yin SS. Quality of life after radiofrequency ablation combined with transcatheter arterial chemoembolization for hepatocellular carcinoma: comparison with transcatheter arterial chemoembolization alone. Qual Life Res. 2007; 16(3):389-97.

8. Yeo W, Mo FK, Koh J, et al. Quality of life is predictive of survival in patients with unresectable hepatocellular carcinoma. Ann Oncol. 2006; 17(7):1083-9.

9. EORTC. EORTC Q1Q C30 [Online]. 2014 [cited 2014 Sep]. Available from http://groups.eortc.be/qol/eortc-qlq-c30.

10. Fayers P, Aaronson NK, Bjordal K, Sullivan M. EORTC QLQ-C30 scoring manual. Brussels: EORTC Publications, 1997.

11. EuroQol. About EQ5D [Online]. 2014 [cited 2014 Sep]. Available from http://www.euroqol.org/about-eq-5d.html.
12. Botega NJ, Bio MR, ZomignaniMA, Garcia C Jr,,Pereira WA. Mood disorders among in-patients in ambulatory and validation of the anxiety and depression scale (HAD). Rev SaudePublica. 1995; 29(5):355-63.

13. Sullivan M, Karlsson J, Ware JE. The Swedish SF-36 Health Survey-I. Evaluation of data quality, scaling assumptions, reliability and construct validity across general populations in Sweden. Soc Sci Med. 1995; 41:1349-58.

14. Ganz PA, Rowland JH, Desmond KA, Meyerowitz BE, Wyatt GE. Life after breast cancer: understanding women's health-related quality of life and sexual functioning. J Clin Oncol. 1998; 16(2):501-14.

15. Zigmond AS, Snaith RP. The hospital anxiety and depression scale. Acta Psychiatr Scand. 1983; 67(6):361-70.

16. Hermann C. International experiences with the Hospital Anxiety and Depression Scale - A review of validation data and clinical results. J Psychosom Res. 1997; 42(1):17-41. 